\title{
Discussion on Fruiter Professional Information Service Mode of Shandong Province
}

\author{
Wang Zhi-jun ${ }^{1,2, *}$, Jiang Meng ${ }^{2}$, and Cheng Shu-han ${ }^{2, * *}$ \\ ${ }^{1}$ College of Horticulture Science and Engineering, Shandong Agricultural University, \\ Taian 271018 China \\ ${ }^{2}$ College of Information Science and Engineering. Shandong Agricultural University, \\ Taian 271018 China \\ \{wzj, shcheng\}@sdau.edu.cn, jiangmengbz@163.com
}

\begin{abstract}
In order to grasp the real status of rural agricultural information services, relying on Fruiter Professional Information Service Mode of Shandong Province, professional information data collection and integration, information service mode, the construction of base and information site, building of expert team and training system are discussed in this paper. The research results have theoretical significance and practical value on the construction of rural informatization demonstration province. It will promote the country to explore the experience of rural agricultural information and patterns.
\end{abstract}

Keywords: professional information, service mode, agricultural informatization.

Shandong province is famous for its apple. The planting area is more than 5 million mu and the production is more than 7.988 million tons[1], accounted for $19.4 \%$ and $26.2 \%$ of the total respectively. The production stands first in China. But the production of apple in Shandong province is at the stage of decentralized management based on farmers whose comprehensive quality is not high. Therefore the information level of apple industrial technology is kept at a low level and the scale of apple processing enterprises other than concentration juice production is limited. Compared with standardized production, Standardization of processing and information management, there is still a long way to go. It's necessary to construct fruiter professional information service system, lengthen the Apple industrial chain in order to breaking through the Production bottleneck, innovating techniques of cultivation management and improving the fruit quality of apple.

In this paper, a bridge is built among farmers, production and marketing enterprises, buying and selling fruit and processing units, government authorities and scientific and technical personnel to provide integrated service for apple production, management and decision-making. It helps employees adapt to the complex and fast-changing market discipline, improve their scientific management and decision-making level in preproduction, production and post-production. It can also promote specialization,

\footnotetext{
* The main interesting research topics are computer networks and agriculture informationization.

** Corresponding author.
} 
standardization and informatization of the apple industry and achieve the goals of increasing farmers' income, enhancing benefits of enterprises and promoting the new rural construction.

\section{Professional Information Data Collection and Integration}

\subsection{Data Collection}

\section{(1) Achieving the information of orchard through the sensor network}

We set up all kinds of sensors in orchard. According to different needs we can choose different data collection methods, such as real-time information collection, manual information collection and timing information collection. In order to provide basis to apple precision management decision-making consultation systems such as apple disease-carrying insect harms management decision-making consultation system precisely, the tree structure evaluation and regulation decision support system, orchard ecological environment dynamic assessment system meteorological information, growth dynamic information, plant diseases and insect pests occurrence information, and soil nutrient and water stress information will also be gathered timely and automatically from various sources of information.

\section{(2) Through the science and technology literature query system}

With the help of domestic and foreign large-scale agricultural science and technology literature database introduced by Shandong Agricultural University and other colleges and universities, such as Wanfang Data Service Platform, Zhonghong Database, PQDT, Tsinghua Tongfang Chinese periodical database and CABI Database, we can provide updated knowledge domain for agricultural management and agricultural science and technology personnel.

\section{(3) Obtaining information timely through intelligent search engine}

Developing stronger intelligent search engine, searching information resources on the internet timely and loading into the local database, we will complete to update information in time.

\subsection{Data Integration}

During the application of the developed program, we could select, review, edit and transport the collected information automatically to the content management module through the data provider, and finally realize the automatic storage operation of the data collection.

Considering the variability of the information selected-it covered diversified messages such as information on agricultural production, agricultural research, business management, cultural life, vocational education and so on, and it also included multiple media types like the text, audio, video, images, animation, ppt, etc- we unified the scientificity, popularity, consistency and timeliness of the integrated resources to make it flexible and accessible for the farmers, fulfilling the actual demand 
of them[2]. In the functional design, simple, cross-database information retrieval make it easy for farmers to quickly and easily find relative information of all kinds to solve the practical problems. Navigation systems, decision support and other functions can effectively help increase the production of a farmer, corresponding with the farmer's cognition and habit.

\section{Diversified Services}

In the production of rural information service in the past, we mainly use the traditional media during the information transmission as the supporting platform, aided by the telecommunications network and the CATV network. But in the new service model, we are applying the triple play as an important supporting platform, taking apple professional information service system of the Shandong province as the basis, carrying out the integrated and professional services for the apple industry, providing technical support for the different types of the existing grassroots information services organization, extending their platform to the top of the industry chain, their services to the end of the industry chain. Professional information service apple supplied contains the following services:

\subsection{Information Dissemination Online}

According to data of The Statistics Report Of China Internet Development [3], until the end of December 2012, the scale of China's Internet users reached 564 million, Internet penetration rate reached $42.1 \%$, rural population reached 156 million, accounted for $27.6 \%$ of the China's Internet users, making an increase of approximately 1960 people than the number by the end of the last year. Apple's professional information service platform of Shandong Province makes full use of the Internet platform for all types of users and published a variety of preproduction, production and post-production information for the whole industry chain. Fig.1.

\subsection{Service Hotline of the Spark Science and Technology}

12396 is the public service number promoted by the Ministry of Science and Technology and the Ministry of Industry to the unify of the country's rural science and technology information. It's a platform aims to provide farmers hotline counseling, interactive messaging, remote video and technical training services. Shandong Province is one of the nation's first rural information pilot provinces, and had officially opened 12396 service hotline on December 25, 2008. At present, it has built a provincial information service center as the core, setting Ji'nan, Tai'an, Yantai 11 municipal information service center as the backbone, making 285 basic information service station as the main body, establishing a massively interacting, convenient and efficient 12396 Grass-roots Service system of the spark science and Technology. 


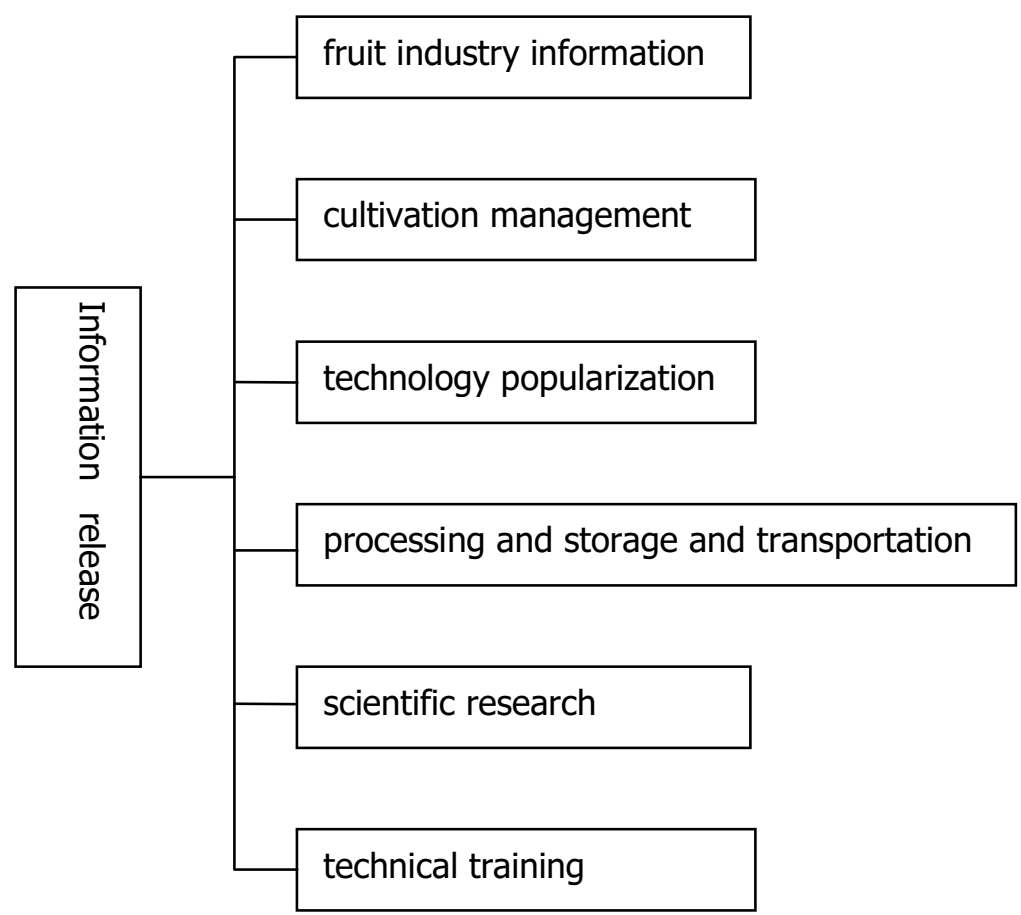

Fig. 1. Information delivery of Industry chain

At present, 12396 call platform is located in the provincial information service center. You can enjoy the automated voice, artificial agents, and three-way calling and other services[4-5], and effective docking with the rural Shandong Radio channels live broadcasts. 12396 has become Radio telephone expert image and has turn to 1 to more service from the 1 to 1 service, and effectively expanded the scope of services and the influence of the platform.

\subsection{Video System}

According to the demand of farmers, we can install video system client in the information service stations, open the remote video system, and organize experts to teach. Also we can provide apple production technology, technical guidance, pest diagnosis of fruit tree, technical training, live video technology, expert lectures and other services online. [6]In order to avoid the high cost, low efficiency and so on by looking for experts solving problem and accepting technical guidance, we provide video\&audio bidirectional answer, network interaction and remote dialogue with them. When communicating with them, specialists could provide the farmers timely and accurate production safety measures and timely technical service, give a guide to the farmers on the scientific management. We could also employ various experts regularly, 
in a irregular or special way to do speeches, online teaching. The mode that direct dialogue between experts and farmers is established to enhance the pertinence and effectiveness of the farmers' work.

\subsection{Short Message Service}

SMS platform developed by Fruiter Professional Information Service platform of Shandong province send the farmers information about cultivation management, technology popularization, policy, market, science and technology, agricultural materials, weather, and product [7]. The information are divided into two categories: public good and value-added services. Some content can be sent to customers free of charge via a push such as government announcements, popularization of policy, agriculture news, outburst epidemic, severe weather warning, market conditions, agricultural science and technology, rich information, labor supply and demand, health control, etc. Others can be sent by charging such as market analysis, professional and technical guidance, exporting statistical information. This kind of SMS platform not only widen the channel of the circulation of the rural information, close communication and interaction between the government and farmers but also motivate the farmers to learn information technology and promote the countryside informatization construction. Satisfying the personalized needs of farmers, growers can gain information anytime and anywhere, enjoy the fast, accurate, economical information service.

\subsection{Expert System}

Agricultural expert system summarizes and collects knowledge and technology in the field of agriculture, large amount of valuable experience of agriculture accumulated by agricultural experts, various information and data and mathematical model obtained from the experiments by using artificial intelligence, knowledge engineering knowledge representation, reasoning, knowledge acquisition technology. Based on the above, a variety of agricultural "computer expert" computer software system with intelligent analysis and reasoning, independent knowledge base which is very convenient to increase and modify the knowledge was established. Developments tools have explanation function and make users do not have to understand the computer programming language. Usual computer program systems cannot come up to it.

At present, the developed expert systems based on apple's professional information service have apple precision management expert system, prevention and control of plant diseases and insect pests' expert system and nutrition diagnosis and recommended fertilization expert system. These systems provide users with decision-making and analysis of the predictive information that have processed and individualized information service and knowledge service. These also provide strong support for the auxiliary production, improving production efficiency, cultivating new type farmers and guiding the agriculture industrial structure adjustment. 


\subsection{Internet Protocol Television Service}

IPTV, Internet protocol television is a manageable multimedia business of Security, interactivity, and the reliability. It contains the Internet, multimedia, communication technologies and delivers TV, video, graphics, and data, etc [8]. IPTV is different from the traditional analog cable TV, also different from classical digital TV. Traditional and classical digital television is unidirectional broadcast , frequency-divided, timing and so on. Although classical digital television has a lot of technical innovation, its signal form is changed. But the transmission mode of media content is not changed.

In this project, the IPTV service platform is built relying on the Shandong unicom company. Farmers can browse Qilu three agriculture information and watch live television program at home using TV by receiving a dedicated set-top box.

\subsection{Mobile Internet}

In recent years, rural informatization construction in China has made great achievements. But effected by computer penetration rate, the network cost and other factors, the implementation of information technology in rural areas has a certain difficulty [9]. Mobile Internet device, characterized by numerous in variety, low cost and convenient access, become the main Internet terminal for rural netizens. It has provided an opportunity for the popularization of the Internet in unenlightened rural areas[10]. ( The Internet development report in rural China,2011 ). The report shows that up to the end of December 2012, the portion of mobile Internet users is increasing, from $69.3 \%$ to $74.5 \%$. And using mobile phones to access the Internet is becoming the main method for rural information service. By the end of 2010,the number of mobile phone users in Shandong has reached 61.9 million (Shandong statistical yearbook in 2011 ) and there are an average of 2.3 mobile phones in each household. The use of $3 \mathrm{G}$ smart phones is rapidly growing with an annual growth rate of $45 \%$. Therefore, we developed the specialized mobile version of apple professional information service system, in order to provide convenience for apple growers to get relevant information by mobile phones.

\section{Apple's Professional Information Base and Site Construction}

Rural information service station is an important content and carrier of the construction and promotion of rural informatization, and it's also the main guiding force and a kind of intermediate link. Rural information service stations are in a key position in the construction of rural information.

By setting up some agricultural informatization demonstration base and choosing growers with representative, stronger driving force, higher character of culture, innovative ideas and enthusiasm as applications demonstration households, we can gradually influence and improve the Information Consciousness of surrounding farmers, expand the radiation area, and promote agricultural informatization from point to area [11]. 
Take different varieties, different regions, different kinds of soil and orchard, tree-age, different technology and other factors into consideration during the process of project implementation. Now we have already built base stations and pilot sites in many places, such as Qixia, Penglai, Rizhao, Mengyin, Yiyuan, Feicheng and Xintai, and received a good demonstration effect.

Through the construction of service stations, we have directly integrated the provincial rural information service platform with rural areas, and reached a flattening goal. During the construction of the sites, we ensured the quality of service by completing the management system and unifying service standards. We also kept exploring the long-term development mechanism by innovating service mode and summarizing experiences.[12]

\section{The Establishment of the Expert Team}

Build a professional service team including technical staffs coming from higher education institutions, scientific research institutions and different government competent departments and growers who has rich planting experiences. They should work and serve all together. The system also cooperates with the Centre for National Apple and Industry Technology Innovation Strategic for Alliance, aiming to guide industrial development and promote technological innovation. We must make full use of interactive video, interactive messaging, telephone communication, remote online messages and all other informational means to answer farmers' production problems accurately and effectively.

\section{$5 \quad$ Full Equipped Training System}

We need to create a training pattern mainly focus on agricultural information training, build some informationalized training bases orienting to "three dimensional rural" and also exploit distinctive training course materials concerning fruit tree planting technique and workforce training skills. We must create a database for planting technique videos, supported by fruit professional information service platform, the rural modern Remote Education System and different kinds of agricultural information sites. With the help of these videos we can provide rich video content and improve farming skills. As to the issues of common concern and new technologies and problems resulting from development of times, we will let farmers take concentrated training, or invite experts to make technical sessions, in order to answer their questions and improve service performance. Carry out training for farmers aiming at rural labor transfer employment and agricultural practical technology application and promotion. We must meet the needs of skills and information service, promote the spiritual and cultural construction in rural areas, and then help farmers to improve ideological and moral standards and also to increase their cultural knowledge and production technology. Speeding up the transformation process from traditional farmers to modern farmers, we will eventually promote the economic development in rural areas. 


\section{Conclusions}

Information Technology is the trend of economic and social development in the world, the rapid development of information technology and full penetration of China's agricultural and rural economic development provides a new opportunity[13,14]. This study aims to build a new type of rural agricultural information service system, to establish a long-term mechanism of agricultural information service in rural area, promote the combination of technology and industrial advantages, improve the level of rural information service, promote the development of modern agriculture and urban and rural areas as a whole and to promote rural agricultural informationization to explore the experience and mode.

\section{References}

1. Statistical Yearbook of Shandong (2011)

2. Zheng, G.H.-I., et al.: Study on the Agricultural Information Service Innovation and its Practice Exploration. Journal of Anhui Agri. Sci. 35(36), 12128-12129 (2007)

3. Statistical report on Internet development in China

4. Wang, Z.-L., Zhang, G.-Q.: The present situation and countermeasure research of rural informatization in Shandong Province. Anhui Science \& Technology (2012)

5. Jing, D.-D., Duan, L.-J.: Discussion on the operation mode of Agricultural Science andTechnology Information Service Platform—-takeing Hunan Spark Science and Technology 12396 Information Service Platform as an example. Technological Development of Enterprise 7, 9-11 (2010)

6. Cai, S.-H., Xu, N.: The Implementation of Quaternity Information Model of Rural Area in Hebei Province. Journal of Hebei Agricultural Sciences 5, 103-105 (2011)

7. Chi, X.-Q.: Discussion on mobile phone SMS platform and promotion of agricultural information in China. Journal of Anhui. Agri. Sci. 18, 310-313 (2006)

8. Yang, W.-N., Zhao, J.: Analysis and design of IPTV technology based on P2P network television. Information Systems Engineering 11, 45 (2012)

9. Xue, F., Zhang, L.-Y.: Discussion on the rural information technology brace model based on cheap mobile phone. Science \& Technology Progress and Policy 24, 89-91 (2010)

10. Rural China Internet Development Report of 2011 (2011)

11. Zhang, J.-P.: Discusses the development pattern of China's rural agricultural information service. China Agricultural Information 5, 14-17 (2011)

12. Li, X.-F., Lu, W.-L.: National progress and analysis of rural informationization demonstration province construction. China Science and Technology Achievements 13 (2012)

13. Fang, G.-Z.: Constructing the New Mode about Agriculture Information Service of Supply and the Demand-Based on Apart Areas of Shandong. Chinese Agricultural Science Bulletin 28(26), 291-297 (2012)

14. Zhao, J.: Research on Agricultural Informatization in and Abroad. Document; Information \& Knowledge 6, 82-87 (2007) 